\title{
Revisión de Literatura Sobre Anormalidades Neurobiológicas en la Esquizofrenia
}

\author{
Jorge Arturo Sebiani Moreira \\ Universidad de Iberoamérica
}

\section{Resumen}

Se realiza una revisión bibliográfica actualizada sobre las anormalidades neurobiológicas en la esquizofrenia. Se investiga utilizando las bases de datos de Google Scholar y EBSCO-host. La evidencia sugiere que la esquizofrenia es en su centro una patología de hipo-conectividad entre redes neuronales que se representa en anormalidades neuroanatómicas, tanto regionales como a nivel celular. Esto es apoyado por estudios de neuroimagen, encefalografía, estudios postmortem, estudios GWAS y voxelwise.

Palabras clave: Esquizofrenia, Neurobiología, Revisión;

\begin{abstract}
A literature review was done about the neurobiological abnormalities in schizophrenia. The databases used to obtain the information were Google Scholar and EBSCO-host. The evidence points towards schizophrenia being a pathology of hypoconnectivity between several neural networks at its core. This is represented in neuroanatomical abnormalities, both at a regional and cellular levels. This conclusion is supported by neuroimaging, encephalography, postmortem, Genome-wide association, and voxelwise studies.
\end{abstract}

Key Words: Schizophrenia, Neurobiology, Review 
La esquizofrenia es una enfermedad mental de severidad variable que depende de una gran cantidad de componentes biopsicosociales y del curso de la enfermedad misma, siendo que aún bajo el cumplimiento de los predictores de buen pronóstico, resulta ser impredecible. Según Torrey (2019) el pronóstico de la enfermedad se puede conceptualizar en una regla de tercios; un $30 \%$ se recupera completamente, otro 30\% tiene síntomas crónicos en remisión, otro 30\% es resistente al tratamiento y el $10 \%$ restante muere por suicidio.

En cuanto a la prevalencia, en una revisión sistemática por Charlson et al. (2018), se identifica una tasa global de $0.28 \%$ con esquizofrenia, si bien el porcentaje es variable de país a país. En dicha revisión no se encuentran diferencias entre sexos y la mayoría de los casos inician durante la adolescencia tardía y la adultez temprana. El número total de casos resulta desproporcionadamente elevado en el continente asiático; un fenómeno que se atribuye al rápido crecimiento poblacional durante las últimas 3 décadas.

Los síntomas y criterios diagnósticos de la enfermedad según el DSM-5 y el CIE-10 incluyen síntomas positivos, negativos, cognitivos y conductuales (Asociación Psiquiátrica Americana, 2013; Organización Mundial de la Salud, 1993) que se detallan en la Tabla 1.

En cuanto a la historia del estudio neuroanatómico de la esquizofrenia, antes del final de la década de los años setenta los cerebros de las personas con esquizofrenia eran analizados a través de estudios postmortem en los cuales los investigadores no encontraban ninguna diferencia aparente en la composición o morfología del tejido cerebral en las personas que habían sido diagnosticadas (Dunlap, 1924; Rosenthal y Bigelow, 1972).

Esto cambió en 1967 con la tomografía computarizada, un método de escaneo cerebral. Ya para el año 1973 se habían realizado más de 3 millones de escaneos cerebrales en el mundo (Sociedad internacional de tomografía computarizada, 2018). Si bien la resolución obtenida de las imágenes era pobre, resultaba en una importante herramienta para el estudio del cerebro. Pronto surgió el interés en estudiar el cerebro de las personas con esquizofrenia por tomografía computarizada. 


\section{Tabla 1}

Síntomas de la Esquizofrenia según el DSM-5 y el CIE-10 (American Psychiatric Association, 2013; Organización Mundial de la Salud, 1993)

\begin{tabular}{ccc}
\hline Clasificación & DSM-5 & CIE-10 \\
\hline $\begin{array}{c}\text { Síntomas } \\
\text { positivos }\end{array}$ & & \\
\hline & $\begin{array}{c}\text { Alucinaciones } \\
\text { Delirios }\end{array}$ & $\begin{array}{c}\text { Alucinaciones } \\
\text { Delirios }\end{array}$ \\
\hline Síntomas & & \\
Negativos & Expresión afectiva disminuida & Afecto aplanado \\
& Abulia & Abulia \\
\hline
\end{tabular}

\section{Síntomas}

Cognitivos

Eco de pensamientos

Inserción de pensamientos

Dificultades para pensar

Comportamiento

\section{Catatonia}

Discurso desorganizado (incoherencia)

Comportamiento desorganizado

\section{Catatonia}

Distorsiones en la producción de lenguaje

Comportamiento desorganizado

Nota: El DSM-5 no tiene clasificaciones por tipos de la enfermedad, el CIE-10 conceptualiza la esquizofrenia por: paranoide, hebefrenia o desorganizada y catatónica.

Los resultados de estos estudios estremecieron el campo académico, en el tanto se encontró que algunas personas con esquizofrenia contaban con ventrículos más voluminosos, fenómeno que hoy se conoce como agrandamiento ventricular (o ventricular enlargement) (Golden et al., 1980; Weinberger et al., 1979; Weinberger 1980).

Progresivamente se desarrollaron metodologías de estudio a través de escaneos cerebrales, y se estableció que el agrandamiento ventricular está asociado a un menor volumen de materia gris y materia blanca; siendo uno de los fenómenos más concurrentes en escaneos cerebrales de personas con esquizofrenia (Nickl-Jockschat, y Abel, 2016; Svancer, y Spaniel, 2021). 
Otro avance en el estudio de la neurobiología consiste del primer antipsicótico, la Clorpromazina, un fármaco que a la fecha estaba siendo estudiado como analgésico y para el cual se encuentra por coincidencia una serie de efectos antipsicóticos en personas con esquizofrenia, reduciendo los síntomas positivos y el comportamiento desorganizado. Luego, en 1975, Seeman et al. realizaron un estudio para determinar la ruta de acción del antipsicótico Haloperidol, encontrando que este grupo de medicinas actúa en los receptores D2 de dopamina, un fenómeno que también es soportado por modelos animales en donde dichos receptores albergarían una mayor concentración de metabolitos de la dopamina sin afectar las concentraciones del mismo neurotransmisor (Madras, 2013; Gründer y Cumming, 2016).

Esto contribuyó a lo que posteriormente se llamó la hipótesis de la dopamina, que consiste en que la hiperactividad del neurotransmisor de dopamina (específicamente el receptor D2) en la vía mesolímbica es una de las variables con mayor peso en la incidencia de la esquizofrenia. Incluso se logró determinar que, para obtener beneficios antipsicóticos, una medicina debe enlazarse con al menos un 65\% de los receptores D2 (Madras, 2013; Gründer y Cumming, 2016).

Ahora bien, el objetivo del presente artículo es realizar una búsqueda sobre la evidencia de investigaciones científicas recientes acerca de las anormalidades neurobiológicas presentes en personas con esquizofrenia. Esto se ha conceptualizado como anormalidades en neuroanatomía, en neurotransmisión y en redes neuronales. Se considera que la etiología de estas tendencias en los cerebros de personas con esquizofrenia es misteriosa, pero es probable que se deban a factores genéticos, epigenéticos, físicos, psicológicos y ambientales (Owen et al., 2016).

\section{Anormalidades Neuroestructurales}

La información obtenida sobre las diferencias en las estructuras cerebrales de las personas con esquizofrenia son recolectadas a través de estudios de neuroimagen, especialmente de resonancias magnéticas. Se citan cinco artículos relevantes para el análisis del fenómeno; un metaanálisis por Haijma et al. (2013), en donde se muestran las estructuras cerebrales con mayor diferencia promedio en comparación al grupo control; La revisión narrativa de Svancer y Spaniel (2021) que analiza la evidencia obtenida del agrandamiento ventricular; Un meta-análisis de 246 estudios por Kuo y Pogue-Geile (2019) que discute sobre la variabilidad entre los volúmenes de las mismas estructuras cerebrales encontradas por Haijma et al. (2013), comparando entre controles y entre personas con esquizofrenia; Finalmente, una revisión por Dietsche et al. (2017) que buscó investigar los cambios estructurales durante diferentes etapas de la enfermedad. 
De estos estudios, se encuentra que los hallazgos más consistentes en la literatura son el agradamiento ventricular y la reducción del volumen en regiones cerebrales (Haijma et al., 2013). Sin embargo, como apuntan Kuo y Pogue-Geile (2019), la variabilidad en volúmenes regionales entre personas con esquizofrenia es alta, y sugieren que se debe a la amplia gama de presentaciones y sintomatología que tiene la psicosis, esto puede delimitar diferentes fenotipos importantes de la enfermedad, incluyendo funcionamiento cognitivo, síntomas y curso de la enfermedad.

Las estructuras que mostraron mayor diferencia promedio entre las personas con esquizofrenia en comparación con el grupo control: volumen intracraneal, volumen total cerebral, ventrículos laterales, tercer ventrículo, materia gris total, materia gris del lóbulo frontal, materia gris del lóbulo prefrontal, materia gris del lóbulo temporal, hipocampo, planum temporale, materia gris del giro temporal superior, giro fusiforme y la ínsula (Haijma et al., 2013).

Lo que Kuo y Pogue-Geile (2019) encontraron es que el volumen ventricular demuestra el mayor tamaño de efecto promedio de las estructuras examinadas. También encontraron menor volumen estructural de manera global y en las estructuras previamente mencionadas. Un análisis importante que realizó este estudio, es que los autores utilizaron el núcleo caudado como estructura de control, con el fin de observar si las diferencias se podían atribuir a otro factor o si estas estructuras están propiamente afectadas por la enfermedad. No encontraron diferencias en el tamaño promedio del núcleo caudado entre personas con esquizofrenia y el grupo saludable.

Entre los hallazgos más interesantes del estudio de Kuo y Pogue-Geile (2019), se encuentra que la variabilidad en el volumen estructural de algunas regiones en personas con esquizofrenia es sumamente alta; por ejemplo, los ventrículos laterales tienen $8.7 \%$ de variabilidad, mientras que el tercer ventrículo tiene un $14.1 \%$ más variabilidad entre la muestra al compararlo con el grupo de personas saludables. El volumen intracraneal fue $2.8 \%$ más variable en personas con esquizofrenia. Es importante resaltar que la variabilidad de las otras estructuras mencionadas no es significativa, encontrando una variabilidad similar entre el grupo con la enfermedad y el grupo sin la enfermedad.

Kuo y Pogue-Geile (2019) tuvieron dificultad para explicar la baja variabilidad entre las estructuras a pesar de una diferencia promedio en el volumen de las estructuras (se encuentra que el volumen de las estructuras es poco variable a pesar de tener diferencias promedio entre las personas con esquizofrenia y el grupo control), arrojan la hipótesis de que el efecto de la reducción en el tamaño es igual en todas las personas afectadas, sin embargo eso es poco probable, en cambio, sugieren que se necesita más información sobre la distribución de los volúmenes de las estructuras, tanto en personas saludables, como en personas con esquizofrenia, para generar un entendimiento más concreto del fenómeno. 
Para continuar, la revisión de Dietsche et al. (2017) indica que el componente neurodegenerativo de la esquizofrenia aún no se ha concretado con certeza. Se tiene la impresión de que en personas con mayor riesgo de desarrollar la enfermedad existe menor volumen en la corteza frontal y temporal; volumen que disminuye en el primer episodio y continúa disminuyendo con la cronicidad de la enfermedad. Sin embargo, se sugiere que estos resultados deben ser interpretados con cautela, debido a que existen estudios que no reportan este efecto. Lo mismo ocurre con predictores del curso de la enfermedad, los estudios longitudinales de neuroimagen demuestran resultados mixtos, algunos indicando una correlación entre mal curso de la enfermedad y reducción progresiva, mientras que otros no.

Por lo tanto, estudios en la variabilidad de las estructuras más que en la reducción promedio, dan mejores pistas de lo que puede estar sucediendo. Se debe tomar en cuenta que estos datos son relativamente recientes y que la mayoría de estudios de neuroimagen tienen muestras pequeñas, por lo que los meta-análisis como el de Haijma et al. (2013), son una buena base pasa obtener información. También, se encuentra que la variabilidad en las estructuras no puede ser explicada por factores sociodemográficos, de curso de la enfermedad, o de consumo de antipsicóticos, por lo que se debe investigar más el fenómeno a nivel individual que a nivel grupal (Kuo y Pogue-Geile, 2019).

Para concluir con este apartado, existen cambios estructurales en personas que tienen esquizofrenia en comparación con la población saludable; específicamente ventrículos agrandados y una reducción de la materia gris a nivel global. La variabilidad entre las otras estructuras mencionadas no demostró ser significativa, aunque aún existe una diferencia promedio importante entre estas estructuras en los grupos con esquizofrenia y las personas sin la enfermedad.

\section{Anormalidades Neuromoleculares}

Para continuar, la siguiente sección es sobre neurotransmisión implicada en la psicosis. En las últimas dos décadas ha habido un cambio radical en la conceptualización de la esquizofrenia. Ahora se conoce que la hipótesis de dopamina era tal vez una explicación sobresimplificada del funcionamiento cerebral de las personas con psicosis. Ahora se discute sobre otras anormalidades tanto a nivel molecular como de rutas neuronales, no sólo en la dopamina, sino también en la serotonina, el glutamato y el GABA (Schoonover et al., 2020; Stahl, 2018). 
Stahl (2018) resalta que la hipótesis de dopamina es una de tres hipótesis primarias que se han estudiado. Otra hipótesis popular es la de glutamato, que propone que la hipoactividad en el receptor NMDA en la corteza prefrontal puede incidir en la psicosis. La otra hipótesis es la de la serotonina, donde se discute que la serotonina cortical o la hiperfunción 5-HT2A también puede resultar en psicosis. Esto se conoce por modelos farmacológicos (de estudios en personas saludables expuestas a drogas psicoactivas) y por comorbilidades con otras enfermedades como las demencias y el Parkinson, donde la psicosis se trata con antagonistas de serotonina.

Ahora bien, como apunta Stahl (2018), es probable que estas tres hipótesis tengan un tamaño de efecto importante en la generación de la psicosis que varía de caso a caso, dependiendo de una multitud de variables, tanto genéticas como ambientales. Por ejemplo, se sugiere que la hipoactividad del receptor NMDA puede causar una reacción adversa de sobreproducción de dopamina en la ruta mesolímbica.

Gründer y Cumming (2016) también apuntan al hecho de que aproximadamente $25 \%$ de las personas que tienen la enfermedad no responden al tratamiento con antipsicóticos. Análisis de ocupación de receptores de dopamina usando escaneos PET y SPECT demuestran que los antipsicóticos están ocupando los receptores D2 en el estriado en ambos grupos (los que responden al tratamiento y los que no responden al tratamiento), lo que sugiere que hay algo detrás de la hipótesis de dopamina que regula la respuesta al tratamiento.

Otro factor importante que discuten Gründer y Cumming (2016) es la competencia entre diversos químicos por ligarse a los receptores de dopamina, y que en estudios en donde se han administrado anfetaminas a personas con esquizofrenia con el fin de exacerbar la sintomatología psicótica, se ha concluido que la "labilidad" en la disponibilidad de los receptores D2/3 a tonos alterados de dopamina parece ser un marcador más apropiado para la psicosis. Estos estudios sugieren que existe una heterogeneidad neuroquímica en los receptores de dopamina de las personas con esquizofrenia, dando a entender que pueden existir etiologías, o incluso marcadores biológicos distintos dependiendo de la presentación neurobiológica de la persona; y que están relacionados a la producción, metabolismo y recepción de la dopamina en el estriado.

A esto se le suman las anormalidades en la neurotransmisión de glutamato y serotonina en la corteza prefrontal, el lóbulo temporal y los ganglios basales, que como se mencionó antes, puede tener efectos en la regulación de los niveles de dopamina en la ruta mesolímbica. La hipótesis de este funcionamiento, como es descrita por Stahl (2018), es que la hiperactividad de serotonina o 5-HT2A causa una cascada de glutamato, y que algunas de las proyecciones de estas células activa el área ventral tegmental (punto inicial de la ruta mesolímbica). 
Por otra parte, la densidad postsináptica es el conjunto de proteínas y moléculas que se encuentran en la neurona postsináptica, que junto con el número de espinas de dendritas que son el número de extensiones del axón para crear otras conexiones y la expresión genética de la densidad postsináptica se conocen como los elementos postsinápticos. Berdenis van Berlekom et al. (2019) encontraron que los elementos postsinápticos en estudios postmortem de cerebros de personas con esquizofrenia tienen por lo general una disminución, especialmente en tejido cortical y no subcortical, específicamente la densidad de espinas de las dendritas, con una disminución particular en la corteza prefrontal en la tercera capa cortical (Banerjee et al, 2016).

La densidad postsináptica es uno de los aspectos alterados en la esquizofrenia, en las que alteraciones en proteínas que envían señales y que no envían señales afectan la fuerza de la sinapsis, la plasticidad, y la conectividad. A esto se le suma los componentes intracelulares como los organelos de las células y el citoesqueleto que pueden afectar en la presentación del número y tamaño de las espinas de las dendritas, así como en su funcionamiento (Banerjee et al., 2016).

Devor et al., (2017) sugieren que la esquizofrenia es en parte una enfermedad de excitabilidad de las neuronas, especialmente en los neurotransmisores de dopamina, GABA, glutamato, serotonina, acetilcolina y opiáceos. Esto es apoyado por evidencia transcriptómica de los procesos que integran la neurotransmisión "lenta" y "rápida", que converge en la proteína DARPP-32.

Para concluir con este apartado, se sugiere que la hipótesis de dopamina no cubre todos los puntos necesarios para establecer una conclusión precisa sobre la etiología de la esquizofrenia. Sino que existen otros neurotransmisores asociados, como la serotonina, el glutamato, y el GABA; por lo tanto, se requiere mayor investigación sobre estas rutas moleculares en los cerebros de personas con esquizofrenia para tener soluciones concretas sobre el comportamiento de la enfermedad en la población. Devor et al. (2017) sugieren que la esquizofrenia es un trastorno "asociativo", es decir, una descomposición de la comunicación entre diferentes sistemas de neurotransmisión rápidos y lentos a través de vías de señales intracelulares, y esto puede unificar muchas hipótesis sobre la etiología de la enfermedad.

En especial, se sugiere que los futuros estudios se pueden enfocar en las bases moleculares de la esquizofrenia afuera de la vía mesolímbica y la conectividad con esta ruta, dando a entender que la acción de los neurotransmisores en otras áreas del cerebro (frontal y temporal) también son importantes en su etiología. A pesar de que la acción de que la ruta mesocorticolímbica debe ser entendida de mejor manera para llegar a conclusiones más certeras sobre la presentación de la enfermedad. También, estudios profundos a nivel celular e intracelular pueden dar pistas sobre la etiología particular del fenotipo. 


\section{Anormalidades en Redes Neuronales}

De la evidencia recolectada de electroencefalogramas y magneto encefalogramas destaca el hecho de que las personas con esquizofrenia tienen una P50 (potencial evocado que ocurre aproximadamente 50ms después de un estímulo auditivo) que está suprimida. También existe una falta de inhibición al medir el PPI (respuesta de alerta a estímulo condicionado), y esto se comparte a lo largo del espectro de la esquizofrenia. Así mismo, el MMN, que es cuando se presenta una serie de estímulos auditivos repetitivos seguido por uno inesperado es anormal en la esquizofrenia, presentando una inhibición de la reacción normal ante el estímulo novedoso. Esta evidencia se ha combinado con la metodología de resonancia magnética y se sugiere que se relaciona a disrupciones en la lateralización de los hemisferios cerebrales y procesamiento temporal; dando evidencia que apoya que la corteza auditiva está comprometida en la esquizofrenia (Neustadter et al., 2016).

Neustadter et al., (2016) también resumen que las anormalidades en P300, un medidor de atención seguida por un estímulo novedoso es posiblemente el marcador mejor estudiado y que los déficits en la respuesta de las personas con esquizofrenia y son el mejor biomarcador de la enfermedad a través de electroencefalografía. Varios metaanálisis han encontrado amplitud reducida y latencia prolongada de las hondas cerebrales en personas con esquizofrenia en esta prueba. Se han encontrado lazos genéticos a esta respuesta, incluso se observa en familiares primarios saludables de personas con esquizofrenia.

Así mismo, los autores resumen el rol de las oscilaciones en ondas cerebrales en personas con esquizofrenia; las oscilaciones trabajan en la sincronización de actividad neuronal y son parte crucial del funcionamiento saludable de las redes neuronales. Las ondas de alta frecuencia (beta y gama) apoyan el funcionamiento local y cortical, mientras que las oscilaciones de frecuencia baja (theta y alfa) apoyan el funcionamiento a larga distancia. Las oscilaciones de gama son de importante relevancia en la esquizofrenia porque demuestran una variedad de procesos sensoriales y cognitivos, como ligar características perceptuales, atención selectiva, prominencia de estímulos, memoria de trabajo y función ejecutiva; los cuales son disfuncionales en el fenotipo de la esquizofrenia (Neustadter et al., 2016).

La actividad gama en personas con esquizofrenia va en línea con hipótesis de hiperexcitabilidad local, relacionado a los síntomas positivos (alucinaciones y distorsión de la realidad). Mientras que la reducción de actividad gama se relaciona a pobreza psicomotora, desorganización y síntomas negativos en regiones selectas; estas anormalidades en las oscilaciones gama pueden resultar de disfunciones anatómicas o en sistemas de neurotransmisión (Neustadter et al., 2016). 
Para continuar, la evidencia en cuanto a la conectividad entre regiones cerebrales en la esquizofrenia demuestra una hipo-conectividad en la red auditiva (ínsula izquierda), red central (core network), red de modo default (default mode network), red autoreferencial, la red somatomotora, red afectiva, red de atención ventral, red talámica, red somatosensorial y la red de procesamiento de prominencias. Se sugiere que la red de desorganización o falta de conexión entre las redes neuronales a nivel global tiene suficiente evidencia empírica para apoyar la hipótesis de que la esquizofrenia es un trastorno de déficits en la conectividad neuronal. Incluso existe evidencia apoyando el hecho de que la red de procesamiento de prominencias tiene un rol central en la enfermedad con su comunicación desbalanceada con otras redes funcionales que puede ser la verdadera razón de las dificultades que las personas con esquizofrenia tienen para diferenciar entre representaciones de sí mismo (mundo interno) y el procesamiento de prominencias ambientales (mundo externo) (Davatzikos y Koutsouleris, 2016; Li et al., 2019; Dong et al., 2017; Liu et al., 2018;)

\section{Conclusión}

La esquizofrenia es una enfermedad altamente heterogénea en su etiología, debido a que la enfermedad puede resultar de varios componentes etiológicos en diferentes individuos. Sin embargo, al mismo tiempo se puede clasificar en fenotipos que se comparten entre varios individuos, por lo que Banerjee et al. (2016) sugieren que existe una manera misteriosa en la que estos componentes etiológicos se manifiestan en fenotipos comunes. Una explicación es que estos rasgos etiológicos de alguna forma encuentran caminos finales similares, dando así un fenotipo común. Esta convergencia puede ocurrir a varios niveles, como en los genes, epigenética, proteínas, caminos neuronales, y/o interacciones célula-célula.

Estos rasgos etiológicos posteriormente se manifiestan en niveles emergentes superiores, como en el funcionamiento de las vías neuronales y el tamaño de las estructuras cerebrales; siendo estos los rasgos fenotípicos de la enfermedad, además de lo observable en la conducta, la percepción y el pensamiento de las personas que sufren de la enfermedad.

Rujescu y Giegling (2016) sugieren que el tratamiento de la enfermedad va a cambiar en el futuro para lograr encontrar el antipsicótico más efectivo para la persona. Actualmente esto se basa en impresión clínica sin tomar en cuenta predictores de biomarcadores y perfiles farmacogenéticos. 
También, como sugieren Davatzikos y Koutsouleris (2016), de la evidencia recabada hasta el momento sobre la esquizofrenia y otras enfermedades psiquiátricas, es posible que en el futuro se conceptualicen las enfermedades mentales por los marcadores neurobiológicos que se presentan en las personas. Dado a que la esquizofrenia comparte rasgos neurobiológicos y genéticos con el trastorno bipolar y la depresión unipolar; además de la disfuncionalidad en la conectividad de redes neuronales, Davatzikos y Koutsouleris (2016), sugieren que estas enfermedades son mejor conceptualizadas bajo la sombrilla de "dismetría cognitiva" en vez de la nosología clínica utilizada en la actualidad.

\section{Referencias}

American Psychiatric Association. (2013). Diagnostic and statistical manual of mental disorders (5th ed.). https://doi.org/10.1176/appi.books.9780890425596

Berdenis van Berlekom, A., Muflihah, C. H., Snijders, G. J., MacGillavry, H. D., Middeldorp, J., Hol, E. M., Kahn, R. S., \& de Witte, L. D. (2019). Synapse pathology in schizophrenia: A meta-analysis of postsynaptic elements in Postmortem Brain Studies. Schizophrenia Bulletin, 46(2), 374-386. https://doi.org/10.1093/schbul/sbz060

Banerjee, A., Borgmann-Winter, K. E., Ray, R., \&amp; Hahn, C.-G. (2016). The PSD: a microdomain for converging molecular abnormalities in schizophrenia. In T. Abel \&amp; T. Nickl-Jockschat (Eds.), Neurobiology of schizophrenia (pp. 125-147). essay, Elsevier/Academic Press.

Charlson, F. J., Ferrari, A. J., Santomauro, D. F., Diminic, S., Stockings, E., Scott, J. G., McGrath, J. J., \& Whiteford, H. A. (2018). Global epidemiology and burden of Schizophrenia: Findings from the Global burden of Disease Study 2016. Schizophrenia Bulletin, 44(6), 1195-1203. https://doi.org/10.1093/schbul/sby058

Davatzikos, C., \&amp; Koutsouleris, N. (2016). Computational neuroanatomy of schizophrenia. In T. Abel \&amp; T. Nickl-Jockschat (Eds.), Neurobiology of schizophrenia (pp. 263-282). essay, Elsevier/Academic Press.

Devor, A., Andreassen, O. A., Wang, Y., Mäki-Marttunen, T., Smeland, O. B., Fan, C.-C., Schork, A. J., Holland, D., Thompson, W. K., Witoelar, A., Chen, C.-H., Desikan, R. S., McEvoy, L. K., Djurovic, S., Greengard, P., Svenningsson, P., Einevoll, G. T., \& Dale, A. M. (2017). Genetic evidence for role of integration of fast and slow neurotransmission in schizophrenia. Molecular Psychiatry, 22(6), 792-801. https://doi.org/10.1038/mp.2017.33 
Dietsche, B., Kircher, T., \& Falkenberg, I. (2017). Structural brain changes in schizophrenia at different stages of the illness: A selective review of longitudinal magnetic resonance imaging studies. The Australian \& New Zealand Journal of Psychiatry, 51(5), 500-508. https://doi.org/10.1177/0004867417699473

Dong, D., Wang, Y., Chang, X., Luo, C., \& Yao, D. (2017). Dysfunction of large-scale brain networks in schizophrenia: A meta-analysis of resting-state functional connectivity. Schizophrenia Bulletin, 44(1), 168-181. https://doi.org/10.1093/schbul/sbx034

Dunlap, C. B. (1924). Dementia præcox. American Journal of Psychiatry, 80(3), 402-421. https://doi.org/10.1176/ajp.80.3.402

Golden, C. J., Graber, B., Moses, J. A., \& Zatz, L. M. (1980). Differentiation of Chronic Schizophrenics with and Without ventricular enlargement by of Lurai-nebraska Neuropsychological Battery. International Journal of Neuroscience, 11(2), 131-138. https://doi.org/10.3109/00207458009150337

Gründer, G y Cumming, P. (2016). The Dopamine Hypothesis of Schizophrenia: Current Status. En T. Abel y T. Nickl-Jockschat (Eds). The Neurobiology of Schizophrenia (1ed., 109-124).

Haijma, S. V., Van Haren, N., Cahn, W., Koolschijn, P. C., Hulshoff Pol, H. E., \& Kahn, R. S. (2013). Brain volumes in schizophrenia: A meta-analysis in over 18000 subjects. Schizophrenia Bulletin, 39(5), 1129-1138. https://doi.org/10.1093/schbul/sbs118

International Society for Computed Tomography. (2018, June 27). Half a century In Ct: How computed tomography has evolved. https://www.isct.org/computed-tomographyblog/2017/2/10/half-a-century-in-ct-how-computed-tomography-has-

evolved\#: :text=In\%201967\%20Sir\%20Godfrey\%20Hounsfield,Laboratories\%20using\%2 $0 \mathrm{x}-$

ray\%20technology.\&text=In\%201971\%20the\%20first\%20patient,publicized $\% 20$ until\%20 a\%20year\%20later.

Kuo, S. S., \& Pogue-Geile, M. F. (2019). Variation in fourteen brain structure volumes in Schizophrenia: A comprehensive meta-analysis of 246 studies. Neuroscience \& Biobehavioral Reviews, 98, 85-94. https://doi.org/10.1016/j.neubiorev.2018.12.030

Madras, B. K. (2013). History of the discovery of the antipsychotic dopamine d2 receptor: A basis for the dopamine hypothesis of schizophrenia. Journal of the History of the Neurosciences, 22(1), 62-78. https://doi.org/10.1080/0964704x.2012.678199

Neustadter, E., Mathiak, K., \& Turetsky, B. I. (2016). Eeg and MEG probes of schizophrenia pathophysiology. En T. Abel y T. Nickl-Jockschat (Eds). The Neurobiology of Schizophrenia (1ed., 213-236) 
Nickl-Jockschat., A. T., \& Abel, T. (Eds.). (2016). Neurobiology of schizophrenia. Elsevier Science Publishing Co.

Li, S., Hu, N., Zhang, W., Tao, B., Dai, J., Gong, Y., Tan, Y., Cai, D., \& Lui, S. (2019). Dysconnectivity of multiple brain networks in schizophrenia: A meta-analysis of restingstate functional connectivity. Frontiers in Psychiatry, 10. https://doi.org/10.3389/fpsyt.2019.00482

Liu, F., Tian, H., Li, J., Li, S., \& Zhuo, C. (2018). Altered voxel-wise gray matter structural brain networks in schizophrenia: Association with Brain Genetic Expression Pattern. Brain Imaging and Behavior, 13(2), 493-502. https://doi.org/10.1007/s11682-018-9880-6

Organización Mundial de la Salud. (1993). The ICD-10 Classification of Mental and Behavioral Disorders. [Genève, Switzerland]

Owen, M. J., Sawa, A., \&amp; Mortensen, P. B. (2016). Schizophrenia. The Lancet, 388(10039), 86-97. https://doi.org/10.1016/s0140-6736(15)01121-6

Torrey, E. F. (2019). Surviving schizophrenia, 7th Edition: A Family Manual. HarperCollins Publishers.

Rosenthal, R., \& Bigelow, L. B. (1972). Quantitative brain measurements in chronic schizophrenia. British Journal of Psychiatry, 121(562), 259-264. https://doi.org/10.1192/bjp.121.3.259

Rujescu, D., \&amp; Giegling, I. (2016). METABOLOMICS OF SCHIZOPHRENIA. In T. Abel \&amp; T. Nickl-Jockschat (Eds.), Neurobiology of schizophrenia (pp. 167-177). essay, Elsevier/Academic Press.

Schoonover, K. E., Dienel, S. J., \& Lewis, D. A. (2020). Prefrontal cortical alterations of glutamate and GABA neurotransmission in Schizophrenia: Insights for rational biomarker development. Biomarkers in Neuropsychiatry, 100015. https://doi.org/10.1016/j.bionps.2020.100015

Stahl, S. M. (2018). Beyond the dopamine hypothesis of schizophrenia to three neural networks of Psychosis: Dopamine, serotonin, and glutamate. CNS Spectrums, 23(3), 187-191. https://doi.org/10.1017/s1092852918001013

Svancer, P., \& Spaniel, F. (2021). Brain ventricular volume changes in schizophrenia. A narrative review. Neuroscience Letters, 759, 136065. https://doi.org/10.1016/j.neulet.2021.136065

Weinberger, D. R., Fuller, T. E., Neophytides, A. N. (1979). Lateral cerebral ventricular enlargement in chronic schizophrenia. Archives of General Psychiatry, 36(7), 735. https://doi.org/10.1001/archpsyc.1979.01780070013001

Weinberger, D. R. (1980). Cerebral ventricular enlargement in chronic schizophrenia. Archives of General Psychiatry, 37(1), 11. https://doi.org/10.1001/archpsyc.1980.01780140013001 